\title{
Wenn es in der Schulter klemmt
}

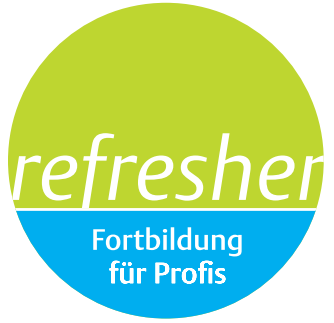

\section{Impingement-Syndrom Blockierende Bewegungseinschränkungen in der Schulter} sind häufig Zeichen eines Impingement-Syndroms. Dabei kommt es zu schmerzhaften Weichteileinklemmungen. Die vielfältigen Ursachen des Syndroms machen eine genaue Befunderhebung und einen darauf abgestimmten Behandlungsplan notwendig.

\section{Lernziele}

$\rightarrow$ Sie kennen die anatomischen Strukturen der Schulter und der Rotatorenmanschette.

$\rightarrow$ Ihnen sind die möglichen Ursachen für ein ImpingementSyndrom bewusst und Sie kennen die Tests, die man bei der Befunderhebung benutzt.

$\rightarrow$ Sie wissen um die Behandlungsmöglichkeiten eines Impingement-Syndroms. der beweglichsten Gelenke und für Verletzungen sehr anfällig. Im Gegensatz zur kleinen Gelenkpfanne ist der Humeruskopf sehr groß. Dass er sich zentral in der Pfanne hält, gewährleistet die Rotatorenmanschette. Zu ihr gehören der M. supraspinatus, der M. infraspinatus, der $M$. teres minor und der M. subscapularis (॰ ABB. 2). Erst das Zusammenspiel dieser Muskeln ermöglicht eine physiologische Bewegung des Armes. Verändert sich etwa die Stellung des Humeruskopfes im Gelenk, kommt es bei bestimmten Bewegungen zu Einklemmungen zwischen dem funktionellen Schulterdach (Fornix humeri) und dem Humeruskopf. Dies geschieht vor allem bei der Abduktion und bei der Flexion im Schultergelenk.
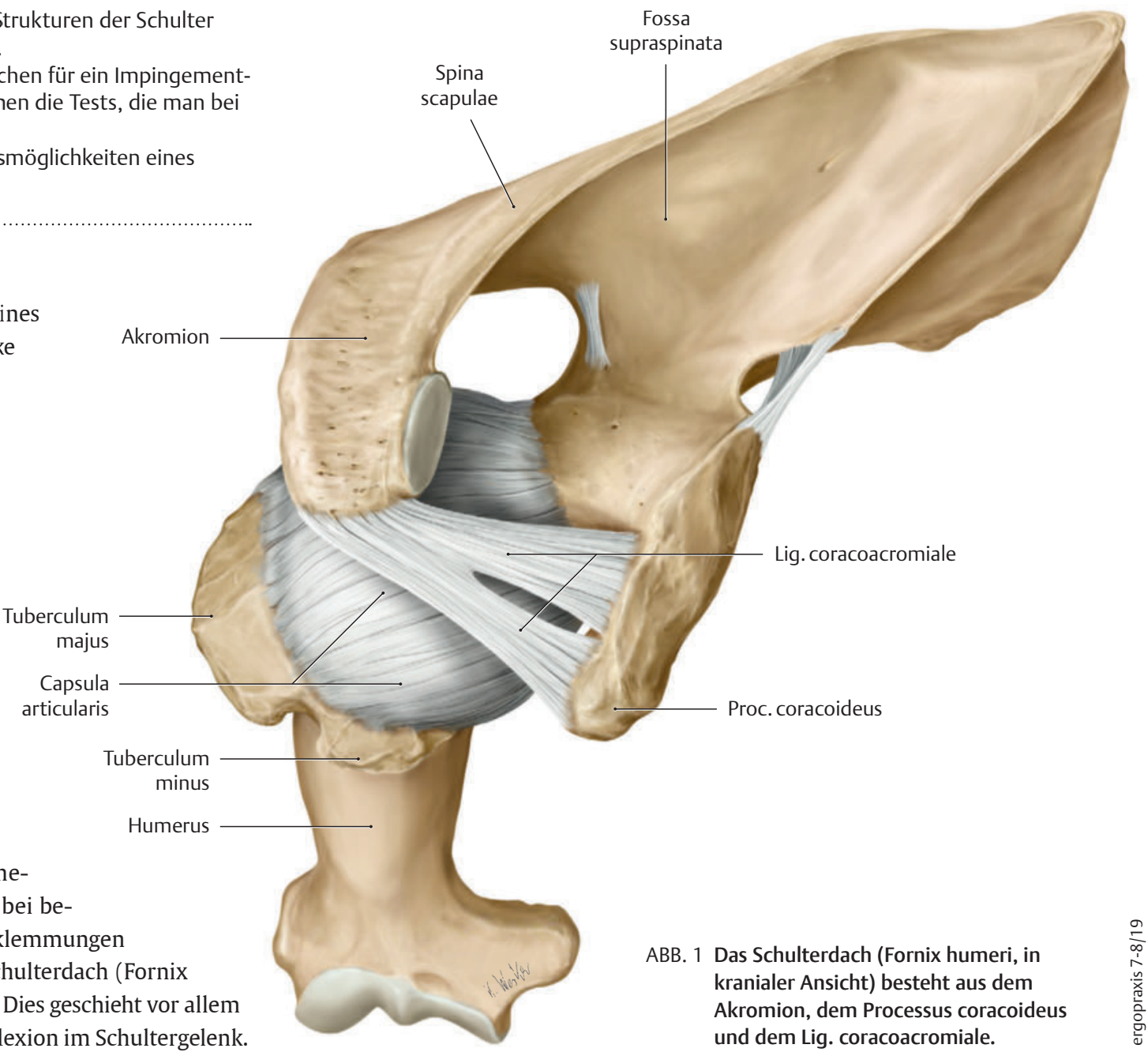


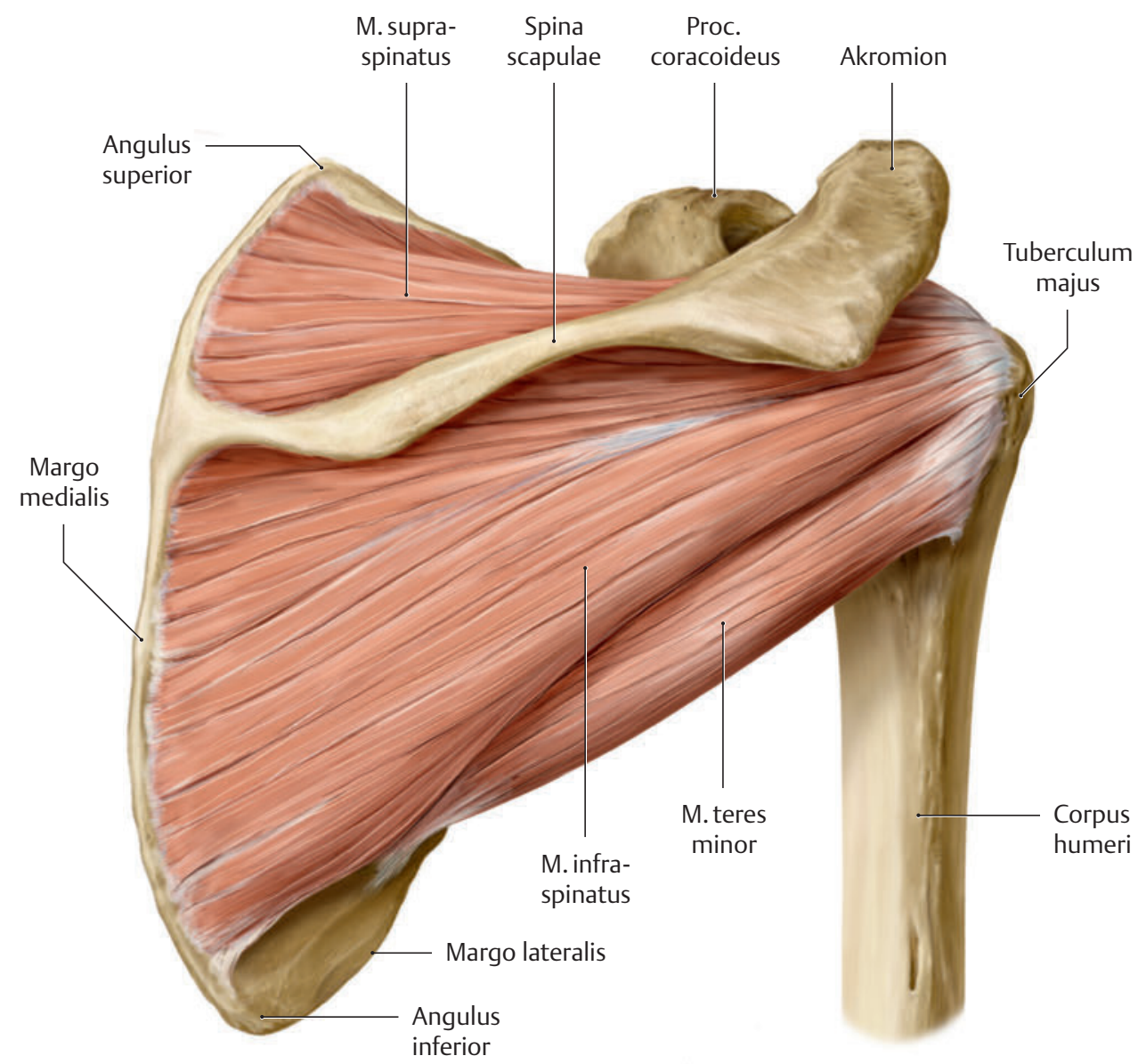

ABB. 2 Zur Rotatorenmanschette gehören der M. supraspinatus, der M. infraspinatus, der M. teres minor und der M. subscapularis. Letzterer ist in dieser dorsalen Ansicht des Schulterblatts nicht zu sehen, da er auf der ventralen Seite des Schulterblattes verläuft.

Anatomische Grundlagen $\rightarrow$ Das Fornix humeri besteht aus dem Akromion, dem Processus coracoideus und dem Lig. coracoacromiale, welches die beiden verbindet ( $\odot$ ABB. 1). Den Bereich zwischen Humeruskopf und Fornix humeri nennt man den subakromialen Gleitraum. Er ist ein unechtes Nebengelenk des Schultergürtels und an allen Bewegungen der Schulter beteiligt. Eine Störung in diesem Gleitlager hat weitreichende Folgen für die Funktionalität des Schultergürtels und die Haltung der Person. Im subakromialen Gleitraum befinden sich die Bursa subacromialis, die Sehne des M. supraspinatus sowie die intraartikulär gelegene lange Bizepssehne. Bei einem Impingement-Syndrom werden diese Strukturen beim Anheben des Armes eingeklemmt, was die typischen Beschwerden hervorruft.

Mögliche Ursachen $\rightarrow$ Es gibt sehr viele unterschiedliche Ursachen, die eine solche Einklemmung hervorrufen können. Strukturelle Veränderungen wie Kalkablagerungen an der Unterseite des Akromions, morphologische Veränderungen des Akromions, eine Bursitis subacromialis oder eine kalzifizierte, entzündete Supraspinatus- sehne können für die Einengung ursächlich sein. Diese führen zu einem sogenannten primären Impingement-Syndrom.

Außerdem ist es möglich, dass Skapulafehlbewegungen, Rumpffehlhaltungen und/oder muskuläre Dysbalancen der Rotatorenmanschette zu einer Dezentrierung des Humeruskopfes führen. Dadurch

\section{g5}

ist das Rotationszentrum des Armes funktionell verschoben. Dies führt wiederum zu Einklemmungen der Weichteile, dem sogenannten sekundären Impingement-Syndrom [1].

Ein möglicher Zusammenhang zwischen den Pathomechanismen des primären und sekundären Impingement-Syndroms wird noch immer kontrovers diskutiert. Heute gehen die meisten Fachleute davon aus, dass sich beide gegenseitig negativ begünstigen [2].

Charakteristische Beschwerden $\rightarrow$ Als typische Beschwerden des Impingement-Syndroms gibt ein Patient andauernde Schmerzen an. Diese treten vor allem bei forcierten Überkopfbewegungen und bei der Abduktion des Armes zwischen $60^{\circ}$ und $120^{\circ}$ verstärkt auf. Dieses Phänomen nennt man „Painful Arc“ (๑ ABB. 8, S. 25). Hebt der Patient den Arm über $120^{\circ}$, verschwinden die Schmerzen in der Regel. 


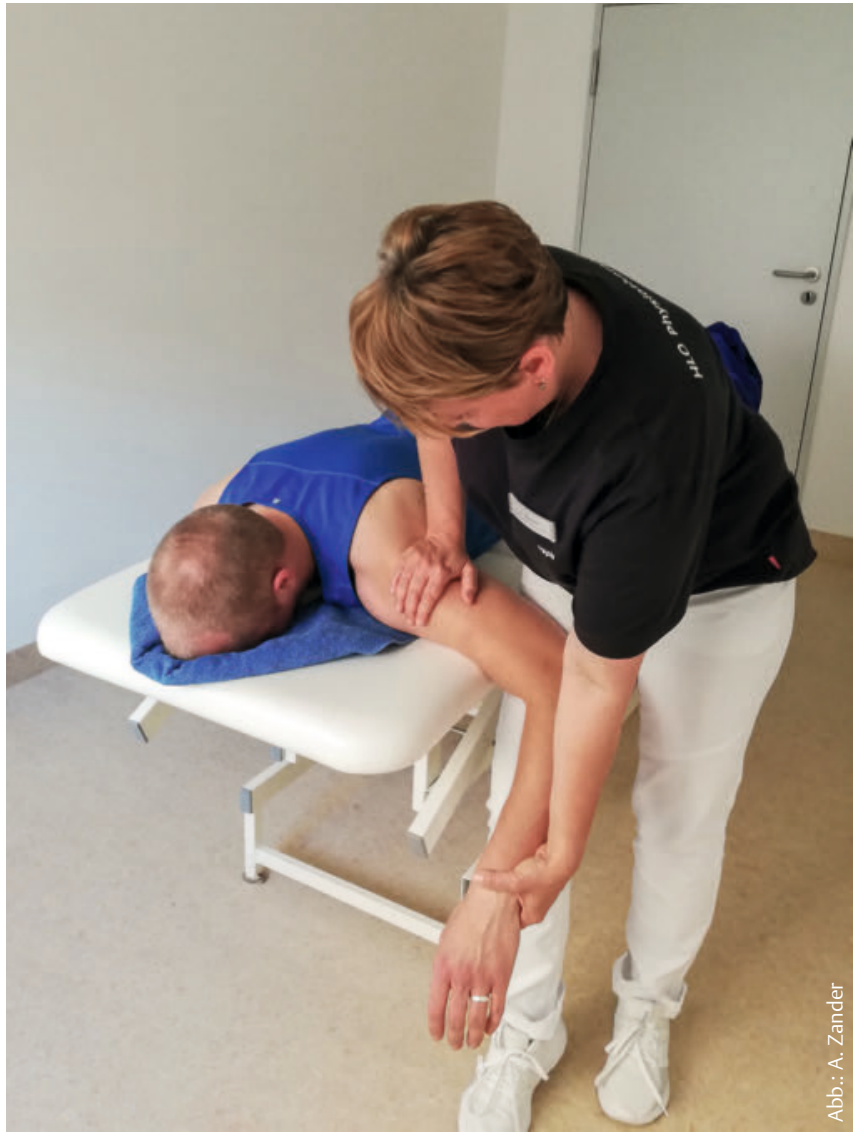

ABB. 3 Mobilisation durch Verntralgleiten des Humeruskopfes bei voreingestellter Außenrotation des Armes: Die Therapeutin stellt den Arm des Patienten in maximal möglicher Außenrotation ein und übt in dieser Position einen alternierenden Schub nach ventral auf den dorsalen Humeruskopf aus, um die Kapsel zu mobilisieren. Diese Übung dient vor allem der Verbesserung der Außenrotation des Schultergelenkes.

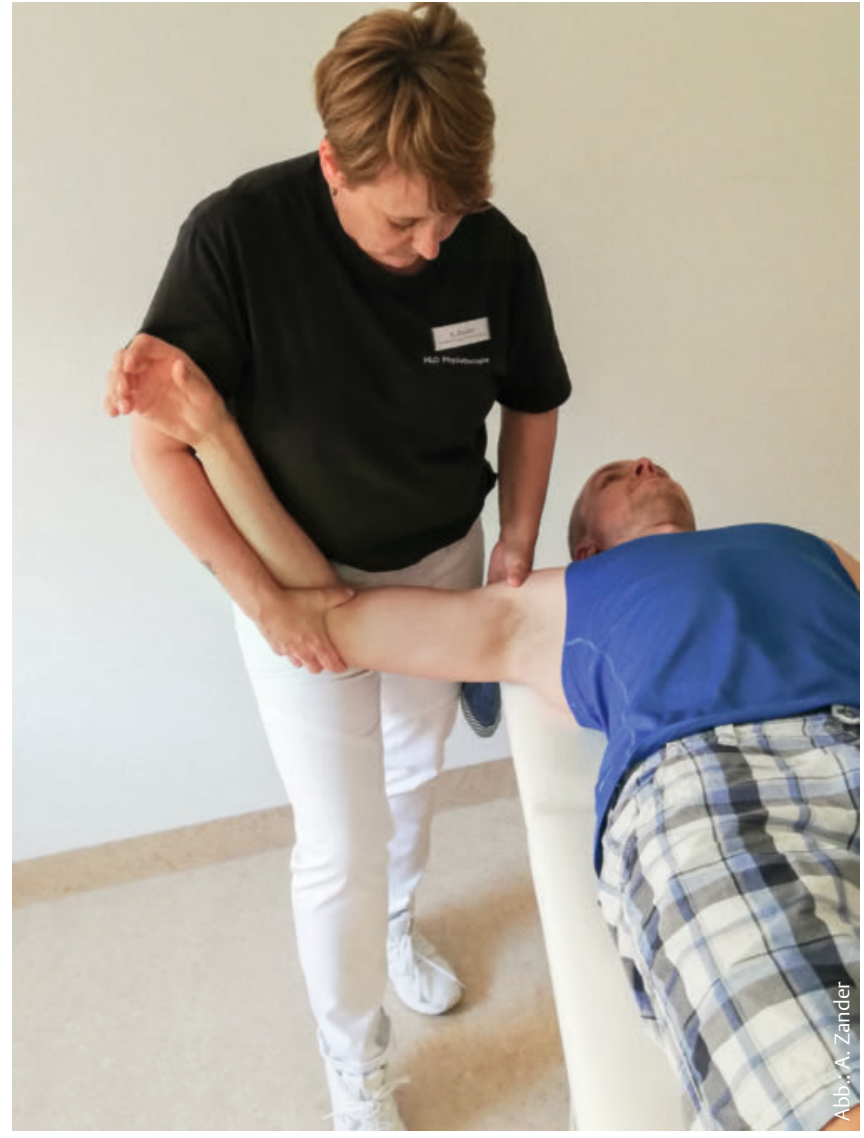

ABB. 4 Mobilisation durch Kaudalgleiten des Humeruskopfes bei voreingestellter Abduktion des Armes: Die Therapeutin stellt den Arm in maximal möglicher Abduktion ein und übt mit ihrer linken Hand einen alternierenden Schubimpuls auf den kranialen Humeruskopf nach kaudal aus, während sie mit ihrer rechten Hand einen Traktionsimpuls für das Schultergelenk gibt. Damit trainiert man insbesondere die Abduktion des Schultergelenkes.

Auf Impingement testen $\rightarrow$ Anamnestisch sollte der Patient neben der gegenwärtigen schmerzhaften Situation auch nach möglichen vergangenen Traumata befragt werden. Impingement-Syndrome können noch Jahre nach einer Verletzung als Zeichen dekompensierter struktureller Veränderungen auftreten. Die klinische Untersuchung umfasst die aktive und passive Überprüfung der Beweglichkeit, isometrische Muskelfunktionstests, die Kraft- und Instabilitätstestung sowie zusätzliche, spezielle Impingement-Tests (๑ TAB., S. 24). Sie ergänzen die Untersuchung und sind in ihrer Gesamtheit unerlässlich, auch wenn sie einzeln betrachtet eine vergleichsweise geringe Spezifität und Sensibilität aufweisen [3].

Bei der passiven Beweglichkeitsüberprüfung ist die Abduktion häufig ohne Schmerzen möglich, die Bewegungen können aber auch schmerzhaft eingeschränkt sein. Eine Schwäche tritt vor allem in der Abduktion und Außenrotation auf und wird mittels isometrischer Muskelfunktionstests festgestellt. Hierbei sollte der oft über ein Schwächegefühl beim Heben des Armes und versuchen über Ausweichbewegungen, den Painful Arc zu umgehen.

Zudem kann man eine veränderte Skapulabewegung (Skapuladyskinesie) beobachten, die als Zeichen der muskulären Dysbalance zu werten ist. Insbesondere beim Senken des Armes (Adduktion) zeigt sich gegen Ende der Bewegung ein Abheben des medialen Skapularandes, wie bei einer Scapula alata („Engelsflügel“). Außerdem ist häufig die Innenrotation mit Adduktion des Schultergelenks schmerzhaft eingeschränkt. Dies kann man überprüfen, indem man den Patienten den Schürzengriff durchführen lässt (Hände hinter dem Rücken wie beim Zubinden einer Schürze zusammenführen). 


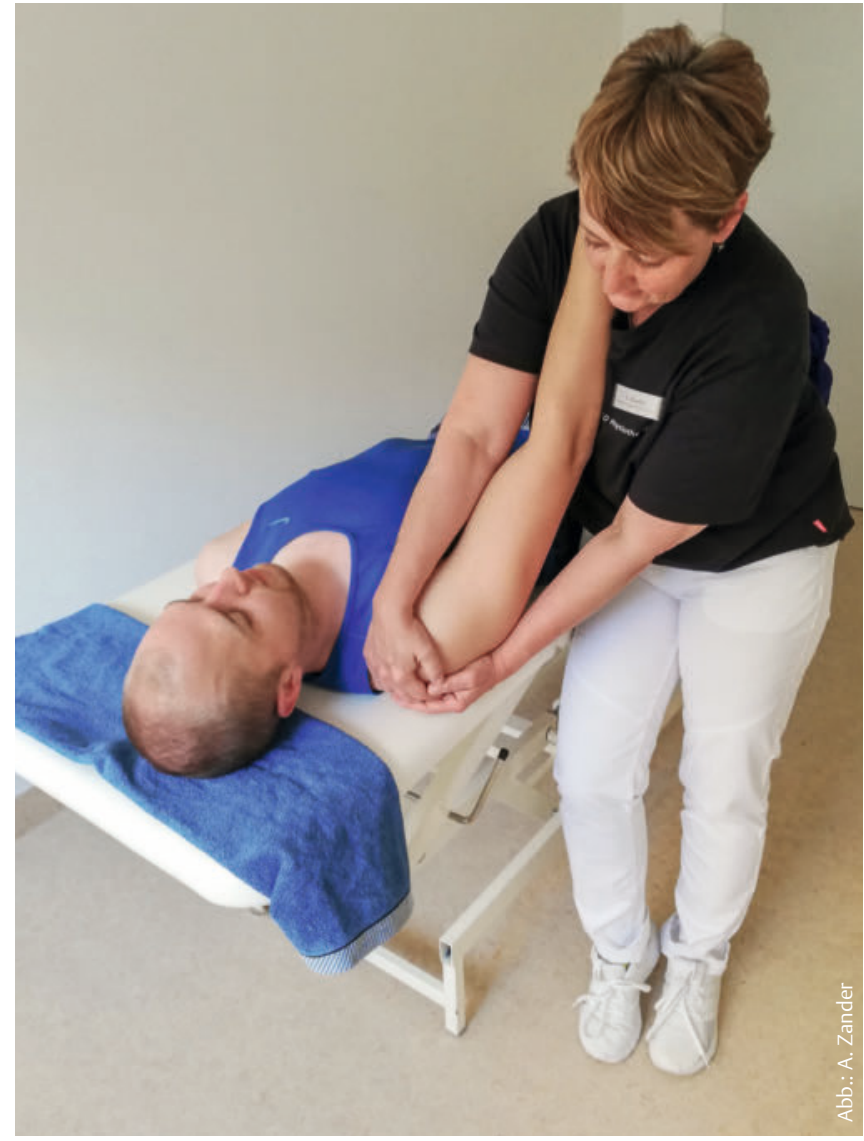

ABB. 5 Isometrische Kräftigung der Schulterblattfixatoren in der geschlossenen Kette: Der Patient spannt die Skapula gegen die Unterlage, indem er mit seiner Hand leicht an der Schulter der Therapeutin zieht. Diese Übung wird im Sinne einer isometrischen Anspannung ausgeführt. Die Therapeutin spürt dabei in ihren Händen einen deutlichen Druck gegen die Unterlage.
Schmerz zu reproduzieren sein. Ist dies nicht der Fall, muss eine nervale Komponente mitbedacht werden und eine neurologische und neurodynamische Untersuchung erfolgen. Führt eine isometrische Abduktion und Außenrotation unter gleichzeitiger Traktion (Auseinanderziehen der Gelenkpartner) zu einer Schmerzentlastung, deutet dies auf eine Affektion der Bursa subacromialis hin, da diese durch die Traktion entlastet wird. Um den Schwächegrad beurteilen zu können, macht die Therapeutin eine Muskelfunktionsprüfung nach Janda.

Schmerzen lindern ist essenziell $\rightarrow$ Das Therapieziel ist neben der Schmerzfreiheit vor allem die Wiederherstellung der Gelenkfunktion und damit der alltagsgerechte Einsatz der Schulter. Eine deutsche Leitlinie zur Behandlung von Impingement-Patienten liegt bislang nicht vor, und insgesamt ist die evidenzbasierte Grundlage für die konservative Therapie sehr überschaubar [3].

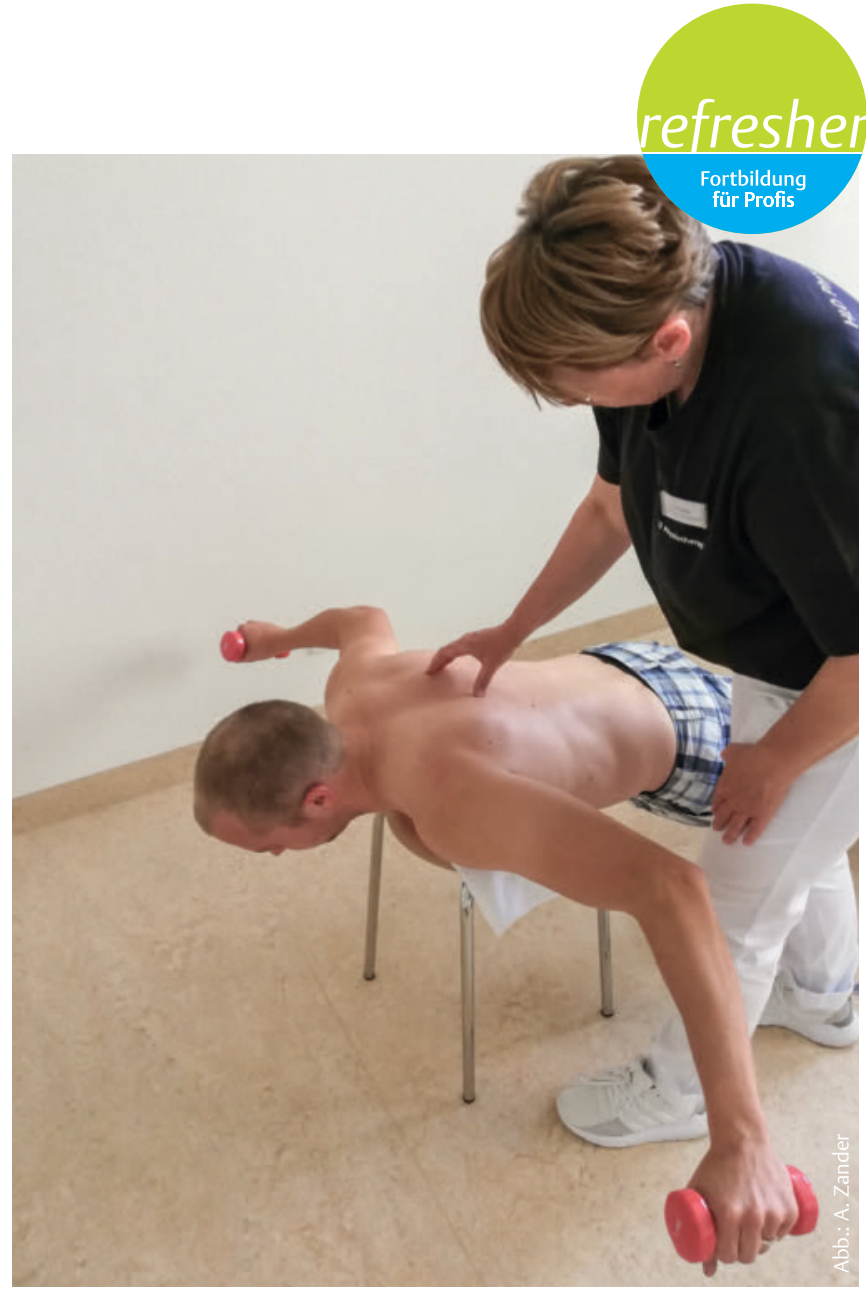

ABB. 6 Kräftigung der Schulterblattfixatoren in der offenen Kette: Der Patient bewegt die Arme mit leicht angewinkelten Ellenbogen nach oben und senkt sie langsam wieder herab (2-3 Durchgänge à 10-12 Wiederholungen). Der Kopf soll in Verlängerung der Wirbelsäule gehalten werden, der Blick des Patienten richtet sich nach unten bzw. leicht schräg nach vorne.
ABB. 7 Muskelaufbau:

Der Patient befindet sich im Vierfüßlerstand und hat ein Theraband um das Handgelenk seines betroffenen Armes gelegt, in diesem Fall der linke Arm. Mit der rechten Hand hält er das Band fest. Dann zieht er das Theraband mit dem linken Arm nach außen und dreht dabei auf dem aufgestellten Ellenbogen den Arm konzentrisch in die Außenrotation. Die Rückbewegung in die Ausgangsstellung erfolgt langsam und exzentrisch. Während der gesamten Übung darf der Patient seine Skapulafixation nicht verlieren.

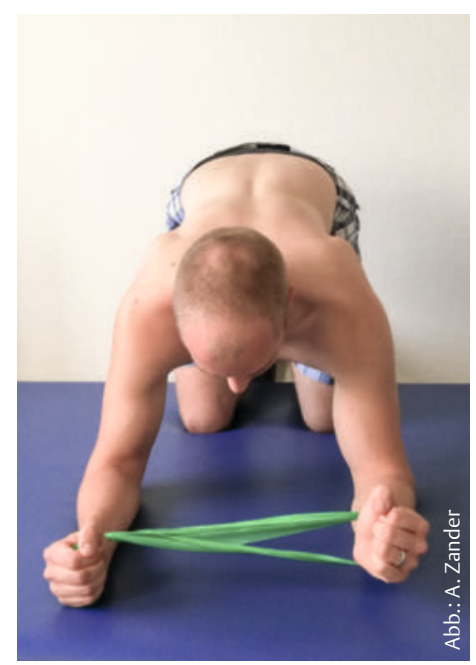




\begin{tabular}{ll} 
Test & Ablauf \\
Painful-Arc-Test $\rightarrow$ & Abduktion des Armes und Reproduktion der Schmerzen zwischen $60^{\circ}$ und $120^{\circ}$ \\
& $\rightarrow$ Absenken des gehobenen Armes führt in diesem Bereich ebenso zu Schmerzen. \\
\hline Hawkins-Test $\rightarrow$ & Reproduktion der Schmerzen ist möglich, wenn das Schultergelenk sowie der Ellenbogen um $90^{\circ}$ flektiert sind und der Arm \\
& maximal innenrotiert ist. \\
$\rightarrow$ & wird hervorgerufen durch eine Einklemmung der Weichteile subakromial zwischen Tuberculum majus und Lig. coracoacromiale \\
\hline Neer-Zeichen $\rightarrow$ & Reproduktion der Schmerzen ist möglich, wenn die Therapeutin den Arm in Flexion und gleichzeitiger Innenrotation führt, \\
& während sie mit der anderen Hand die Skapula fixiert. \\
$\rightarrow$ & wird hervorgerufen durch Anstoßen des Tuberculum majus an das Akromion \\
\hline Jobe-Test & Arm soll in $90^{\circ}$ Abduktion, Innenrotation und 45 Flexion gehalten werden. \\
$\rightarrow$ & Patient wird aufgefordert, den Arm weiter anzuheben bei deutlichem Widerstand gegen die Therapeutin. Dadurch erfolgt eine \\
& Reproduktion der Schmerzen und der Schwäche.
\end{tabular}

TAB. Für das Impingement-Syndrom in der Schulter gibt es spezifische Tests, die man in der Befunderhebung durchführt.

Grundsätzlich sollte die Therapie multimodal aufgestellt sein und sich vor allem nach dem zugrunde liegenden Pathologiemechanismus richten. Multimodal bedeutet in diesem Fall, dass die Therapeutin nicht nur auf der strukturellen lokalen Ebene arbeiten sollte, sondern auch auf segmentalem Level. Das können vegetative Techniken zur Schmerzlinderung an der Brustwirbelsäule sowie Gelenkmobilisationen sein. Ein ADL-Training mit Aufzeigen und Üben von Entlastungsstrategien und eine Hilfsmittelberatung gehören ebenso in ein multimodales Konzept.

Bei Patienten mit einem primären Impingement-Syndrom steht die frühzeitige Gelenkmobilisation im Vordergrund. Bei einem sekundären Impingement-Syndrom hingegen sollte die Skapulastabilisation im Fokus stehen. Diesen beiden Behandlungsansätzen geht stets eine Schmerzbehandlung voraus, bei der die Therapeutin schmerzlindernde Techniken anwenden kann. Dazu gehören beispielsweise passive Techniken wie die heiße Rolle, Massagetechniken für die verspannte Muskulatur oder Triggerpunktbehandlung

Akute Phase: Mobilisation

In der akuten Phase des Impingement-Syndroms sollten die schmerzauslösenden Bewegungen, insbesondere Überkopfbewegungen und Stresspositionen, vermieden und der Arm sollte geschont werden. Die Therapeutin erarbeitet hierfür mit dem Patienten modifizierte Arbeitshaltungen im Sinne eines ADL-Trainings, um die Strukturen zu entlasten. Das könnte sich beispielsweise darin äußern, dass Gegenstände im Arbeitsplatzbereich und im häuslichen Umfeld auf Sichthöhe gelagert werden, um Bewegungen über Kopf zu minimieren. Hinzu kommt das Üben von Alltagsbewegungen mit dem nicht betroffenen Arm, welcher den betroffenen Arm ablösen soll. Passive Bewegungsübungen in der Skapulaebene unter $90^{\circ}$ Abduktion erhalten das Bewegungsausmaß und wirken gleichzeitig schmerzlindernd. In der Skapulaebene finden Bewegungen des
Armes statt, die zwischen $30^{\circ}$ und $45^{\circ}$ vor der Frontalebene erfolgen. So wird das Schultergelenk am wenigsten belastet, und die Kräfte wirken optimal für die Muskelarbeit. Bei Patienten mit einem primären Impingement-Syndrom beginnt die Therapeutin mit der manuellen Gleitmobilisation (॰ ABB. 3 UND 4, S. 22). Bei Patienten mit einem sekundären Impingement-Syndrom erarbeitet die Therapeutin zunächst eine gute Skapulamobilisation und beginnt mit stabilisierenden Übungen (॰ ABB. 5, S. 23).

Zweite Phase: Bewegungsausmaß erweitern

Die zweite Phase der Rehabilitation startet, sobald sich Muskelfunktion und Beweglichkeit verbessert und sich die Schmerzen reduziert haben. Wann das der Fall ist, ist von Patient zu Patient unterschiedlich und daher nur schwer zeitlich einzugrenzen. In diesem Abschnitt der Behandlung steht neben der Erweiterung des Bewegungsausmaßes die Zentrierung des Humeruskopfes und der Kraftaufbau zur Normalisierung des Bewegungsablaufes des gesamten Schultergürtels im Vordergrund. Dies gilt für beide Impingement-Typen.

Die Therapeutin führt alle Maßnahmen der akuten Phase befundorientiert weiter. Neben isometrischen Kräftigungsübungen kommen nun Übungen in der Skapulaebene im geschlossenen System hinzu. Geschlossenes System bedeutet, dass man Muskeln isoliert trainiert, bevor sie wieder in die Muskelkette integriert werden. Das gilt vor allem beim sekundären Impingement-Syndrom. Dabei trainiert der Patient den M. serratus anterior und den M. trapezius pars ascendens. Hierfür steht der Patient aufrecht und hält seinen Arm zunächst in Nullstellung. Dann beugt er ihn im Ellenbogen um $90^{\circ}$ und hält dabei ein Theraband auf leichter Spannung fest. Zieht er nun die Skapula in Richtung Wirbelsäule und hält sie dort, arbeitet er gegen den Widerstand des Therabandes. Dann löst er die Spannung langsam wieder und gibt dem Band nach. Die Mobilisation des M. serratus 


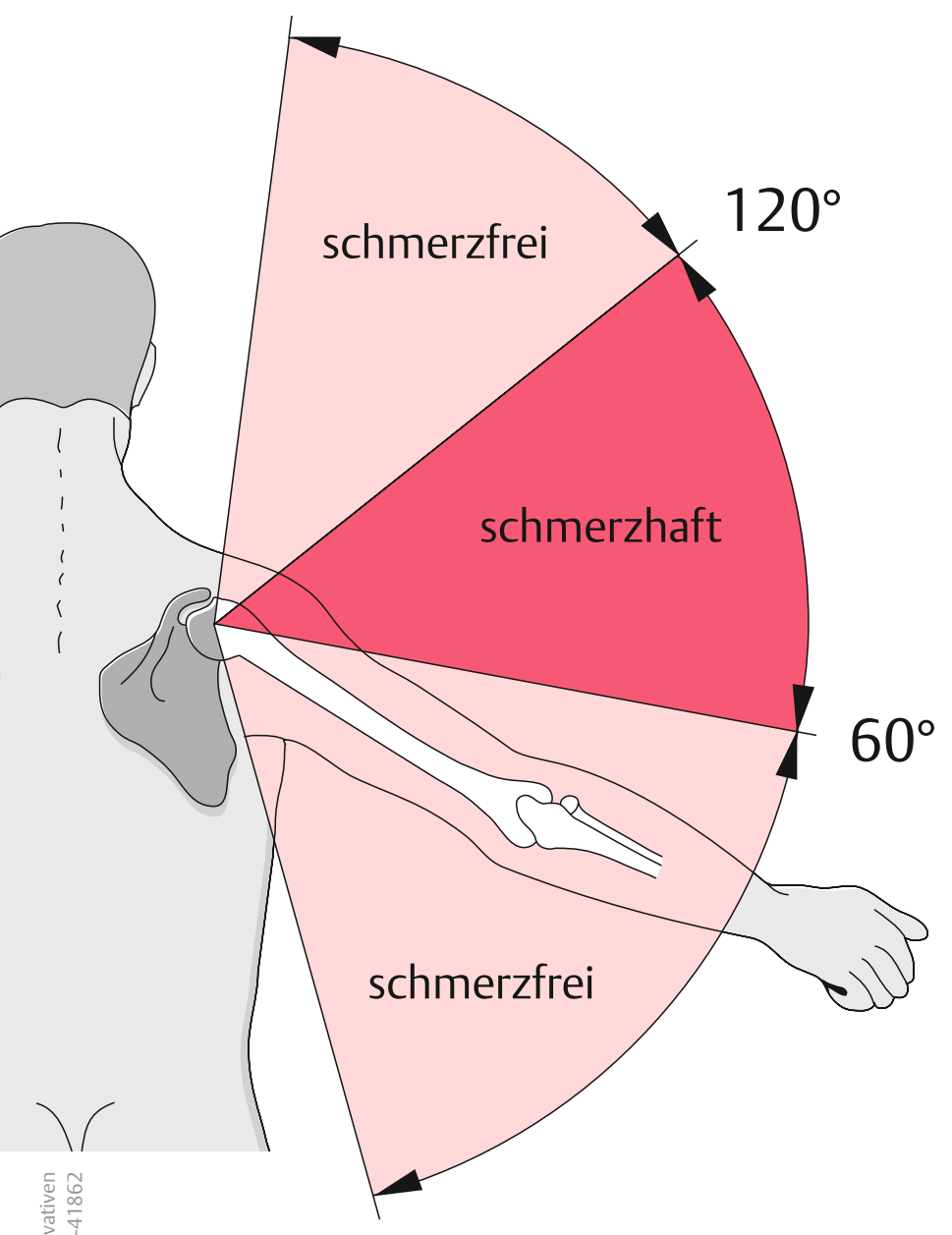

ABB. 8 Beim „Painful Arc“ gibt der Patient bei der Abduktion des Armes zwischen $60^{\circ}$ und $120^{\circ}$ Schmerzen an, darunter und darüber ist er beschwerdefrei.

anterior und des M. trapezius pars ascendens ist zum einen wichtig, da sie das Schulterblatt als Punctum fixum für den Arm stabilisieren. Zum anderen ermöglichen sie das Heben des Arms über $90^{\circ}$. Zudem sollte der Patient in Eigenübungen die schwachen Extensoren der oberen Brustwirbelsäule, die schwachen Außenrotatoren der Schulter und die skapulafixierenden Muskeln trainieren (๑ ABB. 6, S. 23).

Für Personen mit primärem Impingement-Syndrom kommen in dieser Phase zunehmend aktive Dehnübungen zum Einsatz. Sie dienen der Verbesserung der Kapselbeweglichkeit, die schmerzadaptiert auch als Eigenübungen absolviert werden können. Dabei zieht der Patient seinen angewinkelten Arm über die gegenüberliegende Schulter zur Eigendehnung der posterioren Kapselanteile. Ist das schmerzfreie Bewegungsausmaß erreicht, kann man zunehmend mit der konzentrischen/exzentrischen Kräftigung der Innen- und Außenrotatoren beginnen.

\section{Dritte Phase: Muskelaufbau}

In der dritten Phase steht der Muskelaufbau im Zentrum der Behandlung. Diese beginnt, wenn der Patient keine Schmerzen mehr hat, die Skapulafixation ausreichend trainiert wurde und die Muskeln so weit trainiert sind, dass sie wieder in die Muskelkette integriert werden können (๑ ABB. 7, S. 23). Zunehmend sollten nun auch bei ausreichender Skapulafixation diese Übungen im offenen System erfolgen. Offenes System bedeutet, dass der zu trainierende Muskel nicht meht isoliert, son-

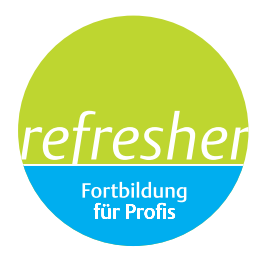
dern in der Muskelkette trainiert wird. Die Kräftigung des M. deltoideus sollte aufgrund seiner humeruskranialisierenden Funktion erst am Ende der Rehabilitation stehen. Ein zu frühes Training reizt erneut die subakromialen Strukturen, was die Entzündung wieder in Gang setzen könnte. Der Muskel kann mittels Theraband oder Hantel trainiert werden, indem der Patient abduzierende Übungen bis $90^{\circ}$ absolviert. Des Weiteren führt er die Kräftigungsübungen für die Extensoren der Brustwirbelsäule weiter. Zusätzlich sollte die Therapeutin dem Patienten Dehnund Mobilisationsübungen für die Brustwirbelsäule zeigen. Eine einfache Übung dafür kann der Katzenbuckel sein, den man aus dem Vierfüßlerstand heraus macht. Wichtig ist, dass der Patient eine übersichtliche, für ihn verständliche Übungsauswahl erlernt, die er mindestens zweimal wöchentlich durchführt. Insgesamt ist in der Regel eine zwei- bis dreimonatige Behandlung notwendig.

Neue Bewegungen in den Alltag integrieren $\rightarrow$ Ein wichtiger Aspekt ist nicht zuletzt auch das Erarbeiten von komplexen Handlungsabläufen mit Bezug zu den alltäglichen Bewegungen und Aufgaben des Patienten. Hier lernt der Patient, Ausweichbewegungen zu vermeiden und Alltagsfunktionen zielorientiert zu üben, um den neuen Bewegungsablauf zu automatisieren. Dazu eignet sich je nach Situation der Einsatz von Gegenständen des alltäglichen Lebens, wie das Anschnallen mit einem Autogurt, bei dem eine schmerzfreie und uneingeschränkte Bewegung der Schulter möglich sein muss.

Die Prognose dieser konservativen Therapie hängt unter anderem von verschiedenen Faktoren ab: der zugrunde liegenden Pathologie, der Dauer der Beschwerden oder der Compliance des Patienten. Bei etwa 60 Prozent der Patienten zeigt sich ein zufriedenstellendes Ergebnis nach zwei Jahren [3]. Die Behandlung eines ImpingementSyndroms erfordert daher von Seiten der Patienten als auch der Therapeuten ein hohes Maß an Geduld und Disziplin. Nur dann wird es irgendwann nicht mehr in der Schulter klemmen.

Andrea Zander

$\rightarrow$ Literaturverzeichnis

www.thieme-connect.de/products/ergopraxis > „Ausgabe 7-8/19“

\section{白 Autorin}

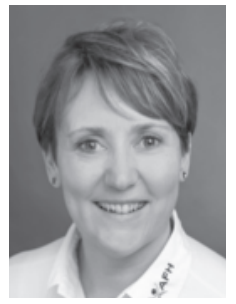

Andrea Zander ist Physiotherapeutin. Seit 1995 arbeitet sie schwerpunktmäßig mit Handpatienten und ist derzeit als fachliche Leitung einer Physiotherapie im Krankenhaus Waldfriede in Berlin beschäftigt. Außerdem ist sie als Honorardozentin bei der Akademie für Handrehabilitation tätig und gibt regelmäßig Fortbildungen für Ergo- und Physiotherapeuten. 


\section{Fragen zum Impingement-Syndrom}

1. Welcher der folgenden Muskeln gehört nicht zur Rotatorenmanschette?
A M. teres minor
B M. subscapularis
C M. infraspinatus
D M. deltoideus
E M. supraspinatus

\section{Wie lautet der lateinische Name für das Schulterdach?}
A Fornix humeri
B Caput coracoacromiale
C Fornix scapulae
D Caput humeri
E Fornix humerale

3. Den Bereich zwischen Humeruskopf und dem Schulterdach bezeichnet man als ...
A ... Sternoklavikulargelenk.
B ... Bursa subacromialis.
C ... Rotatorenmanschette.
D ... Rabenschnabelfortsatz.
E ... subakromialen Gleitraum.

4. Zu welcher Form des Impingement-Syndroms kann eine Rumpffehlhaltung führen?
A tertiäres Impingement-Syndrom
B sekundäres Impingement-Syndrom
C sporadisches Impingement-Syndrom
D primäres Impingement-Syndrom
E basales Impingement-Syndrom

5. Wie nennt man das Phänomen, bei dem Schmerzen im Schultergelenk vor allem bei Überkopfbewegungen und der Abduktion zwischen $60^{\circ}$ und $120^{\circ}$ auftreten?
A Neer-Zeichen
B Painful Move
C Painful Arc
D Sulcus-Zeichen
E Painful Lift

6. Was ist neben Schmerzfreiheit ein weiteres wichtiges Therapieziel in der Behandlung eines ImpingementSyndroms?
A Gelenkfunktion wiederherstellen
B Narben abhärten
C Körperwahrnehmung verbessern
D Kontrakturen vermeiden
E Koordination ausbauen

\section{In der akuten Phase des Syndroms sollte ein Patient ...}
A ... auf jeden Fall einen operativen Eingriff vornehmen lassen.
B ... möglichst viele Schmerztabletten einnehmen.
C ... keine Therapie erhalten.
D ... die schmerzauslösenden Bewegungen vermeiden.
E ... den Arm stets in einem Dreieckstuch lagern.

\section{Wie nennt man das Vorgehen, bei dem man Muskeln zunächst isoliert trainiert, um sie später wieder in die Muskelkette zu integrieren?}
A Übungen im offenen System
B isometrische Übungen
C Übungen im geschlossenen System
D isotonische Übungen
E konzentrische Übungen

9. Was steht in der dritten Phase der Behandlung vor allem im Vordergrund?
A passive Bewegungsübungen
B Schmerzlinderung
C Gleichgewichtsübungen
D ADL-Training
E Muskelaufbau

10. Was können Patienten zu Hause tun, um die Therapiefortschritte zu unterstützen?
A lyengar Yoga
B Dehnübungen mit einem Theraband
C Schulterpolster tragen
D den betroffenen Arm nicht benutzen
E Fitnessvideos anschauen 